University of Nebraska - Lincoln

DigitalCommons@University of Nebraska - Lincoln

February 1996

\title{
Artificial Selection on JHE Activity in Gryllus assimilis: Nature of Activity Differences Between Lines and Effect on JH Binding and Metabolism
}

\author{
Anthony J. Zera \\ University of Nebraska - Lincoln, azera1@unl.edu \\ Jeffrey Sall \\ University of Nebraska - Lincoln \\ Robert Schwartz \\ University of Nebraska - Lincoln
}

Follow this and additional works at: https://digitalcommons.unl.edu/bioscizera

Part of the Microbiology Commons

Zera, Anthony J.; Sall, Jeffrey; and Schwartz, Robert, "Artificial Selection on JHE Activity in Gryllus assimilis: Nature of Activity Differences Between Lines and Effect on JH Binding and Metabolism" (1996). Anthony Zera Publications. 22.

https://digitalcommons.unl.edu/bioscizera/22

This Article is brought to you for free and open access by the Papers in the Biological Sciences at DigitalCommons@University of Nebraska - Lincoln. It has been accepted for inclusion in Anthony Zera Publications by an authorized administrator of DigitalCommons@University of Nebraska - Lincoln. 


\title{
Artificial Selection on JHE Activity in Gryllus assimilis: Nature of Activity Differences Between Lines and Effect on JH Binding and Metabolism
}

\author{
Anthony J. Zera, Jeffrey Sall, and Robert Schwartz \\ School of Biological Sciences, University of Nebraska-Lincoln
}

Genetic lines of the cricket Gryllus assimilis selected for elevated vs. decreased activity of hemolymph juvenile hormone esterase (JHE) exhibited a 6.8-fold difference in enzyme activity by the seventh generation of selection. This documents that hemolymph JHE activity has the capability for rapid evolutionary change. This is the only insect endocrine trait for which such data are currently available. The difference in hemolymph JHE activity between the lines was due, to an equivalent degree, to variation in whole-cricket enzyme activity and allocation of JHE activity to the hemolymph compartment. No differences in kinetic or thermostability characteristics were observed between JHEs from the high- vs. low-selected lines. This suggests that the variation in JHE activity between the lines resulted from selection of variable regulatory genetic factors affecting the synthesis, degradation, or activation of enzyme activity. JHE and JHbinding activity in the hemolymph are genetically correlated, possibly due to co-regulation by the same genetic factors. Finally, in vivo JH degradation was significantly elevated in lines selected for elevated JHE activity. This documents that evolutionary changes in JHE activity alter in vivo $\mathrm{JH}$ metabolism.

Keywords: juvenile hormone, juvenile hormone esterase, selection, JH degradation, Gryllus

Abbreviations: $\mathrm{JH}=$ juvenile hormone; JHE= juvenile hormone esterase; JHE$\mathrm{H}=$ high $\mathrm{JH}$ esterase activity line; JHE-L = low JH esterase line; OTFP = 3 octylthio-1,1,1 -trifluoro-2-propanone.

\section{INTRODUCTION}

During the past three decades a tremendous amount of chemical, biochemical, and physiological data have been obtained on insect endocrine traits (Downer and Laufer, 1983; Kerkut and Gilbert, 1985; Gupta, 1990).However, genetic aspects of insect en- 
docrinology, especially population-genetic aspects, remain poorly studied. Data on the amount, characteristics, and degree of interaction among naturally occurring, genetically based endocrine variations within species have only recently been reported (Wilson and Thurston, 1989; Zera and Tiebel, 1989; Zera and Zhang, 1995; Gu and Zera, 1996). Because of the paucity of population-genetic information, our understanding of the microevolutionary processes that modify or constrain the insect endocrine system is limited.

Artificial selection is a powerful technique for studying the population genetics of phenotypic variation (Arnold, 1987; Falconer, 1989; Garland and Carter, 1994). It allows a direct assessment of the degree to which a trait can evolve. Moreover, the genetically differentiated lines produced by selection can be analyzed to determine the nature of the genetic variation that was present in the original population when selection was initiated. Finally, artificial selection provides important information on genetic correlations between the selected trait and other organismal features. Identifying these correlations is important since they can strongly affect the evolution of the trait under study (Arnold, 1987; Zera and Zhang, 1995).

We are currently in the process of selecting on hemolymph juvenile hormone esterase (JHE) activity in the cricket, Gryllus assimilis (Zera and Zhang, 1995). JHE is an important regulatory enzyme which degrades and regulates the JH titer in several insects (Hammock, 1985; Roe and Venkatesh, 1990). The overall goals of our studies are to determine (1) the amount and characteristics of genetic variation for JHE activity and (2) the extent and nature of genetic correlations between JHE and other endocrine features. In the present study, we focus on the nature of the JHE activity differences between the high and low-selected lines and the consequences of altered JHE activity on whole-organism $\mathrm{JH}$ metabolism and hemolymph $\mathrm{JH}$ binding.

\section{MATERIALS AND METHODS}

\section{Chemicals}

Racemic $\left[10^{-3} \mathrm{H}\right]$ juvenile hormone-III $(12 \mathrm{Ci} / \mathrm{mmol} ; 444 \mathrm{GBq} / \mathrm{mmol})$ was obtained from New England Nuclear (Boston, MA) and OTFP (3-octylthio-1,1,1-trifluoro-2propanone), a transition-state analogue and potent inhibitor of JHE, was a generous gift from Dr. Bruce Hammock, Dept. of Entomology and Environmental Toxicology, University of California at Davis. Other chemicals and solvents used in this study were purchased from Sigma Chemical Co. (St. Louis, MO).

\section{Stocks}

The high- and low-selected JHE activity lines of G. assimilis, investigated in the present study, were the same as those generated and studied by Zera and Zhang (1995). Lines were studied during the seventh through the ninth generations of selection. The protocol used to select for elevated or decreased JHE activity during this study was the same as that used by Zera and Zhang (1995). Briefly, in each selected line, hemolymph JHE activity was measured on 200 individuals on day 3 of the last 
stadium. The fifty individuals with the highest or lowest activity were used as breeders to produce the next generation. A high JHE activity line, designated as "JHE-H," and a low JHE activity line, "JHE-L," were selected in each of three separate trials (= blocks; see Zera and Zhang, 1995). The number following the "JHE-H" or "JHEL" line designation denotes the block to which the line belongs (e.g., JHE-H1 is the high-selected line from block one).

\section{Assays}

In vitro hemolymph JHE specific activity, whole-cricket JHE specific activity, and protein concentration were measured according to Hammock and Sparks (1977), Share and Roe (1988), and Stoschek (bicinchoninic acid method; 1990), respectively. Hemolymph JH-binding activity was measured as described in Gu and Zera (1994) except that racemic rather than [10-R]JH-III was used. In vivo hemolymph volume and JH degradation were measured as described in Zera and Holtmeier (1992) except that crickets were homogenized 45 min rather than 60 min after injection of radiolabeled, racemic JH-III. All experiments were performed on day-3, last stadium crickets.

Experimental conditions for the estimation of Michaelis constants for JH-III, determination of inhibition profiles for OTFP, and determination of thermal denaturation profiles for JHE were the same as those described in Gu and Zera (1994). Only JHE from the high and low selected lines of Block-1 were characterized. For thermal denaturation or inhibition studies, assays were conducted in triplicate at 8 inhibitor concentrations or temperatures. Michaelis constants were estimated using the protocol of Duggleby (1979; see Zera et al., 1992). Ten replicate velocity measurements were performed at 5,000, 250, and $75 \mathrm{nM} \mathrm{JH}-\mathrm{III}$ concentrations. Kinetic parameters and their standard errors were estimated using the non-linear regression program Enzfitter (Leatherbarrow, 1987). Characterizations were preformed on hemolymph from last stadium crickets that were three to seven days old.

\section{RESULTS}

\section{Hemolymph and Whole-Cricket JHE Activities}

Hemolymph JHE activity was significantly higher in the JHE-H vs. JHE-L line in each of the three blocks during the seventh generation of selection (Table 1; $P<0.005$ for $t$-tests in each block). Averaged over the three blocks, hemolymph JHE activity was elevated $6.9 \pm 0.8$-fold in the high vs. low lines. Whole-cricket JHE activity also was significantly elevated in the JHE-H vs. the JHE-L line of each block (Table 1; $P$ $<0.005$ for each of the three $t$-tests). However, the average elevation $(2.4 \pm 0.2$-fold $)$ was significantly less than that of hemolymph JHE specific activity ( $t$-test; $P<0.01$ ).

Within each block, relative hemolymph volume (volume expressed as \% wholebody wet weight) did not differ significantly between the four JHE line/sex classes (e.g., high-males, low-males, etc.; $P>0.1$ in each Kruskal-Wallis test; $\mathrm{N}=4-8$ individuals in each class). Because of the absence of differences among these classes, 
TABLE 1. Hemolymph and Whole Cricket Juvenile Hormone Esterase (JHE) Specific Activity and JH-Binding Activity in High- and Low-Selected Lines of Gryllus assimilis*

\begin{tabular}{|c|c|c|c|c|}
\hline $\begin{array}{l}\text { Block/ } \\
\text { line }\end{array}$ & $\begin{array}{l}\text { Hemolymph } \\
J_{H E^{\mathrm{a}, \mathrm{d}}}\end{array}$ & $\begin{array}{c}\text { Whole-organism } \\
\text { JHE }^{\mathrm{b}, \mathrm{d}}\end{array}$ & $\begin{array}{c}\% \text { JHE in } \\
\text { hemolymph }\end{array}$ & $\begin{array}{c}\text { JH-binding } \\
\text { activity }{ }^{\mathrm{c}, \mathrm{e}}\end{array}$ \\
\hline 1-High & $55.4 \pm 8.6$ & $8.0 \pm 0.9$ & $75.5 \pm 10.7$ & $25.2 \pm 1.6$ \\
\hline 1-Low & $7.9 \pm 0.9$ & $3.3 \pm 0.2$ & $29.6 \pm 3.3$ & $16.1 \pm 0.82$ \\
\hline$H / L$ & 7.0 & 2.4 & 2.6 & \\
\hline 2-High & $53.8 \pm 9.4$ & $10.9 \pm 1.0$ & $68.6 \pm 7.5$ & $87.5 \pm 12.6$ \\
\hline 2-Low & $9.7 \pm 1.8$ & $5.2 \pm 1.1$ & $29.1 \pm 2.0$ & $48.4 \pm 6.8$ \\
\hline $\mathrm{H} / \mathrm{L}$ & 5.5 & 2.1 & 2.4 & 1.8 \\
\hline 3-High & $62.2 \pm 7.7$ & $13.3 \pm 0.3$ & $70.5 \pm 8.7$ & n.d. \\
\hline 3-Low & $7.6 \pm 0.9$ & $4.7 \pm 0.3$ & $24.8 \pm 4.4$ & n.d. \\
\hline $\mathrm{H} / \mathrm{L}$ & 8.2 & 2.8 & 2.8 & \\
\hline \multicolumn{5}{|c|}{ 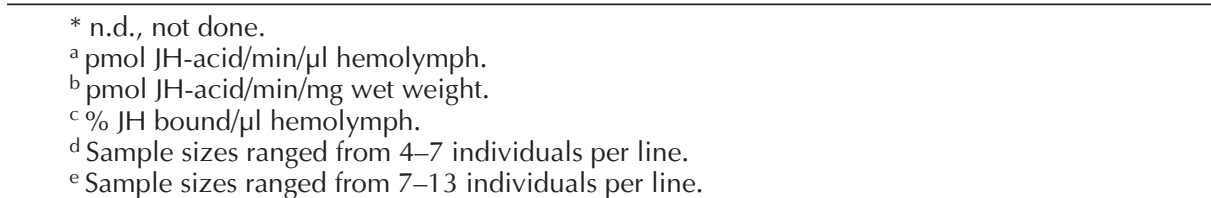 } \\
\hline
\end{tabular}

an overall estimate of relative hemolymph volume was obtained for each block. Values ( \pm standard error) were as follows: $16.5 \pm 0.9 \%(\mathrm{~N}=25 ; \mathrm{Bk}-1), 16.3 \pm 0.9 \%(\mathrm{~N}$ $=30 ; \mathrm{Bk}-2)$ and $15.5 \pm 0.6 \%(\mathrm{~N}=25 ; \mathrm{Bk}-3)$. Using these values and whole cricket and hemolymph JHE activities, the percentage whole-cricket JHE activity in the hemolymph compartment was estimated for individual crickets. Mean \% whole-cricket JHE activity allocated to the hemolymph compartment was 2.4-2.8-fold higher in the JHE-H vs. JHE-L line of each block (Table $1 ; P<0.005$ in each $t$-test). Like the whole-cricket and hemolymph JHE activities, hemolymph JH-binding activity was also significantly higher in the JHE-H vs. JHE-L line in each of the two blocks studied (Table $1 ; P<0.025$ in each Kruskal-Wallis test).

\section{Biochemical Characteristics of JHE}

JH-III Michaelis constants $\left(\mathrm{K}_{\mathrm{M}}\right)$ were $106 \pm 14 \mathrm{nM}$ for JHE-H1 and $133 \pm 15 \mathrm{nM}$ for JHE-L1. These values did not differ significantly ( $t$-test; $P>0.05)$. Thermal denaturation and OTFP-inhibition profiles also exhibited only minor differences between the JHEs from the high and low-selected lines of block-1 (Fig. 1).

\section{In Vivo Degradation of $\mathrm{JH}$ in the Selected Lines}

Paired measurements of JH-degradation and JHE activity in individual crickets from the high and low-selected lines of blocks 1-3 are presented in Figure 2. In these experiments JHE activity was measured on a small hemolymph sample taken just prior to injection of radiolabeled JH-III. Mean percentage JH-III degraded in vivo for the JHE-H and JHE-L lines of the three blocks are as follows: $\mathrm{H} 1(51.1 \pm 4.5 \%, \mathrm{~N}=$ 11), L1 $(29.0 \pm 3.7 \%, \mathrm{~N}=11)$; H2 $(63.5 \pm 2.9 \%, \mathrm{~N}=11), \mathrm{L} 2(47.0 \pm 4.6 \%, \mathrm{~N}=11)$; $\mathrm{H} 3(62.5 \pm 3.8 \%, \mathrm{~N}=12), \mathrm{L} 3(53.5 \pm 2.8 \%, \mathrm{~N}=11)$. These differences were statistically significant between the JHE-H and JHE-L lines of block-1 $(P<0.005)$, block-2 $(P<.025)$, and block-3 $(P<0.05)$ as determined by Kruskal-Wallis tests. 

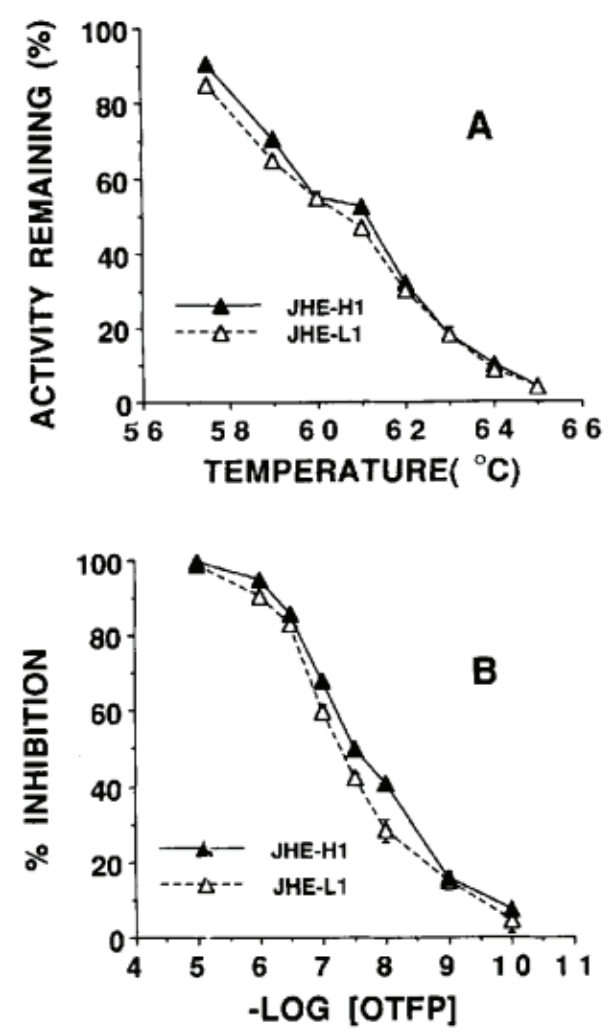

Figure 1. Thermal denaturation (A) and OTFP-inhibition profiles (B) of hemolymph juvenile hormone esterase from the JHE-H1 $(\mathbf{\Delta})$ and JHE-L1 $(\Delta)$ lines. Symbols represent means of three replicates. Bars are standard errors which in some cases are smaller than the symbols. Enzyme was derived from crickets from the ninth generation of selection.

\section{DISCUSSION}

The $6.9 \pm 0.8$-fold divergence in hemolymph JHE activity between JHE-H and JHE-L lines, measured during the seventh generation of selection (Table 1), was greater than the $3.5 \pm$-fold divergence observed during generation 6 (Zera and Zhang, 1995). This indicates that hemolymph JHE activity is continuing to respond to bidirectional selection. Indeed, we have now documented $>10$-fold divergence in hemolymph JHE activity between the JHE-H and JHE-L lines during the 11th generation of selection (Zera, unpublished data). Results of the ongoing selection experiment document that hemolymph JHE activity has the potential for rapid evolutionary change. This is currently the only insect endocrine trait for which such data are available.

We previously speculated that the response to selection on hemolymph JHE activity was due to changes in whole-cricket JHE activity as well as due to changes in the amount of enzyme allocated to the hemolymph compartment (Zera and Zhang, 1995). In the present study we directly estimated the amount of JHE activity in the hemolymph compartment and have documented that altered tissue distribution and altered 

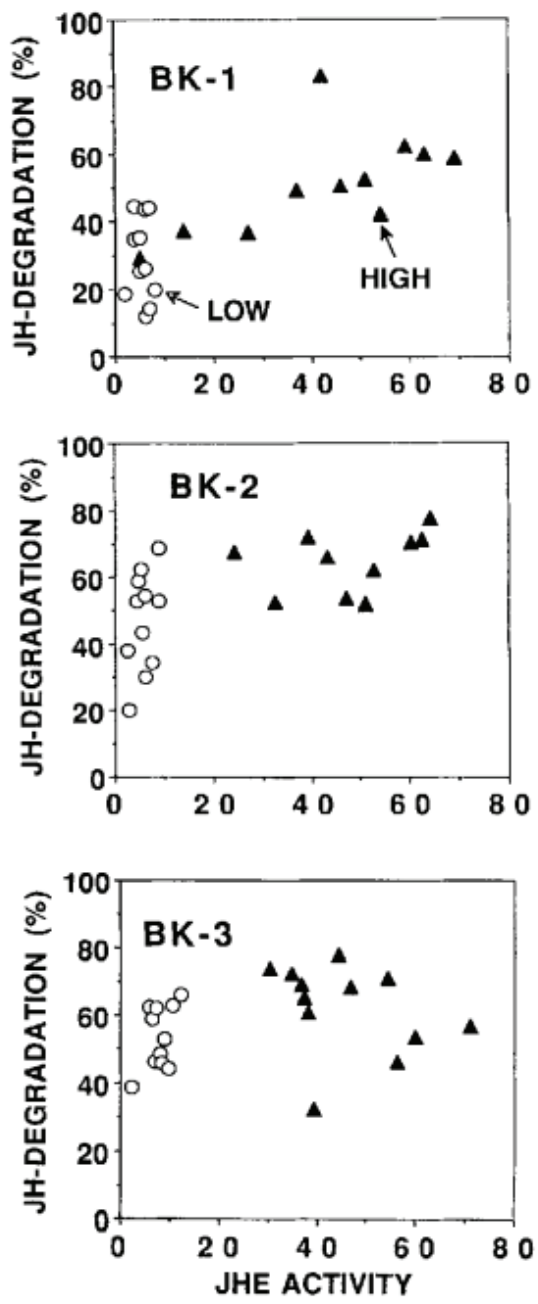

Figure 2. Percentage racemic juvenile hormone-III degraded in vivo relative to hemolymph JHE activity $(\mathrm{nmol} / \mathrm{min} / \mathrm{ml})$ in crickets of the high $(\boldsymbol{\Delta})$ or low $(\mathrm{O}) \mathrm{JHE}$ activity lines from the three blocks ( $\mathrm{BK}=$ block $=$ separate selection trial; see Materials and Methods). Each point represents the measurement of in vivo $\mathrm{JH}$ degradation and in vitro JHE activity in a single cricket. JHE activity was measured in a small hernolymph sample taken just prior to injection of radiolabeled $\mathrm{JH}-\mathrm{III}$. Assays were performed on crickets during the ninth generation of selection.

whole-cricket enzyme activity play an equal role in the response to selection (Table 1). These JHE-selected lines should be very useful for investigating the physiological and molecular mechanisms which regulate the whole-organism and tissue-specific distribution of JHE activity as well as populational variation in these mechanisms. An important goal of our studies is to identify the biochemical basis of the JHE activity differences between the high and low-selected lines. The existence of only small differences between JHE-H1 and JHE-L1 in kinetic and thermostability characteristics indicate that the activity differences between the selected lines are not likely due to 
allozymes or isozymes with altered biochemical properties. Preliminary biochemical data also indicate no substantial differences between JHE from the high and low-selected lines of blocks 2 and 3 (Zera, unpublished data). These results are similar to the minimal biochemical differences between JHEs from high vs. low-activity stocks of the wing-polymorphic congener, Gryllus rubens (Zera et al., 1992). These data point to variation in genetic factors regulating JHE activity (e.g., differential synthesis, degradation, or activation) as being the most likely reason for the activity differences between the high- and low-selected lines. However, we have yet to compare the JHEs with respect to some important kinetic constants, such as turnover number $\left(\mathrm{k}_{\text {cat }}\right)$.

Although genetic correlations may strongly accelerate or retard the evolution of insect endocrine traits, information on their existence is very meager (Zera and Zhang, 1995; Gu and Zera, 1996). Genetic correlations are manifest as indirect responses to selection, that is, by divergence between selected lines for traits not directly selected (Arnold, 1987; Falconer, 1989). We observed such an indirect response to selection for JH-binding activity (Table 1), documenting that it is genetically correlated with hemolymph JHE activity. This observation is consistent with the previous documentation that JHE and JH- binding activity were genetically correlated in half-sib crosses of $G$. assimilis from a stock different from that used in the selection experiment (Gu and Zera, 1996). The correlation between hemolymph JHE and JH-binding activity raises the interesting possibility that these two endocrine features are coregulated by similar variable genetic factors (see "Discussion" in Gu and Zera, 1996). The existence of a genetic correlation between JHE and JH-binding activity contrasts with the absence of a correlation between JHE and JH-epoxide hydrolase activity, the other major JH degrading enzyme (Zera and Zhang, 1995). At present we have no explanation as to why some important endocrine features are correlated with JHE activity while others are not.

Finally, the consistently elevated in vivo JH degradation in JHE-H vs. JHE-L lines in each of the three blocks indicates that JHE activity has been altered sufficiently by selection to affect in vivo JH metabolism. In the previous study of Zera and Zhang (1995) in vivo JH degradation only differed between JHE-H2 and JHE-L2. Since these lines exhibited the greatest divergence in JHE activity, Zera and Zhang (1995) predicted that continued selection for JHE activity should result in each JHE-H line exhibiting an elevated degree of JH degradation relative to its JHE-L line. We have confirmed this prediction in the present study. Besides documenting that JHE activity affects in vivo $\mathrm{JH}$ metabolism, this result indicates that altered JHE activity has the capacity to affect whole-organism traits by affecting the JH titer. We are currently comparing the selected lines with respect to variation in morphological traits, such as flight muscle and wing development, to assess how evolutionary change in endocrine traits underlies the evolution of morphology.

\section{LITERATURE CITED}

Arnold SJ (1987): Genetic correlation and the evolution of physiology. In Feder ME, Bennett AF, Burggren WW, Huey RB (eds): New Directions in Ecological Physiology. Cambridge: Cambridge University Press, pp 189-215. 
Downer RGH, Laufer H (eds): Endocrinology of Insects. New York: Alan R. Liss, Inc.

Duggleby RG (1979): Experimental designs for estimating the kinetic parameters for enzyme-catalyzed reactions. $J$ Theor Biol 81:671-684.

Falconer DS (1989): Introduction to Quantitative Genetics, 3rd ed. New York: Longman Scientific and Technical.

Garland TJ, Carter PA (1994): Evolutionary Physiology. Ann Rev Physiol 56:579-621.

Gu X, Zera AJ (1994): Developmental profiles and characteristics of hemolymph juvenile hormone esterase, general esterase and juvenile hormone binding in the cricket Gryllus assirnilis. Comp Biochem Physiol B Comp Biochem 107:553-560.

Gu X, Zera AJ (1996): Quantitative genetics of juvenile hormone esterase, juvenile hormone binding and general esterase activity in the cricket, Gryllus assimilis. Heredity 76:336-142.

Gupta AP (ed) (1990): Morphogenetic Hormones of Arthropods, vols. 1, 2, and 3. New Brunswick: Rutgers University Press.

Hammock BD (1985): Regulation of the juvenile hormone titer: Degradation. In Kerkut GA, Gilbert LI (eds): Comprehensive Insect Physiology, Biochemistry and Pharmacology, vol. 7. New York: Pergamon Press, pp 431-472.

Hammock BD, Sparks TC (1977): A rapid assay for juvenile hormone esterase activity. Anal Biochem 82:573-579.

Kerkut GA, Gilbert LI (eds) (1985): Comprehensive Insect Biochemistry, Physiology and Pharmacology, vols. 7 and 8, Endocrinology. New York: Pergamon.

Leatherbarrow RJ (1987): Enzfitter. A non-linear regression data analysis program for IBM PC (and true compatibles). Cambridge: Biosoft.

Roe RM, Venkatesh K (1990): Metabolism of juvenile hormones: Degradation and titer regulation. In Gupta AP (ed): Morphogenetic Hormones of Arthropods, vol. 1. New Brunswick: Rutgers University Press, pp 126-179.

Share MR, Roe RM (1988): A partition assay for the simultaneous determination of insect juvenile hormone esterase and epoxide hydrolase activity. Anal Biochem 169:81-88.

Stoschek CM (1990) Quantitation of protein. Methods Enzymol 182:60-63.

Wilson TG, Thurston J (1989): Genetic variation for methoprene resistance in Drosophila melanogaster. J Insect Physiol 34:305-308.

Zera AJ, Holtmeier CL (1992): In vivo and in vitro degradation of juvenile hormone-I11 in presumptive long-winged and short-winged Gryllus rubens. J Insect Physiol 38:61-74.

Zera AJ, Tiebel KC (1989): Differences in juvenile hormone esterase activity between presumptive macropterous and brachypterous Gryllus rubens: Implications for the hormonal regulation of wing polymorphism. J Insect Physiol 35:7-17.

Zera AJ, Zhang C (1995): Evolutionary endocrinology of juvenile hormone esterase in Gryllus assimilis: Direct and correlated responses to selection. Genetics 141:1125-1134.

Zera AJ, Gu X, Zeisset M (1992): Characterization of juvenile hormone esterase from geneticallydetermined wing morphs of the cricket, Gryllus rubens. Insect Biochem Mol Biol 22:829-839. 